After having travelled a short way from the cathode in the vapour jet, the positive ions are stopped and pulled back to the cathode by the impressed electric field. Although most of these positive ions probably disappear by recombinations, many of them will strike the cathode at a point shifted from the cathode spot from which they issued. The impingement on or at least the close approach of large numbers of positive ions to the cathode surface is very probably a necessary and sufficient condition for the formation of a cathode spot. We may therefore expect that these positive ions in the emitted vapour shifted in the magnetic field will cause a new cathode spot to develop, which ultimately will take over all the current from the previous cathode spot, causing the are to move in a more or less discontinuous way in the direction opposite to that which would be expected from elementary electrodynamic considerations.

Research Laboratory,

Westinghouse Elec. \& Mfg. Co.,

East Pittsburgh, Pa., Aug. 1.

\section{Appearance of Noble Gases in Vacuum Tube Discharges.}

The views expressed in the article by A. C. E. in Nature of June 8 , p. 879 , on the appearance of helium in apparatus employed in attempts at atomic synthesis and disintegration are in good accord with results obtained at the Physical Laboratories of the University of Chicago and of Hiram College. A repetition of the work of $R$. W. Riding and E. C. C. Baly, reported in Proc. Roy. Soc., Series A, vol. 109, No. A 749 , p. 186, has been undertaken by me under the direction of Prof. H. B. Lemon of the University of Chicago. The results have been inconclusive on the whole, but in all cases except one, helium has appeared in the observation tubes. The one exception occurred at the end of a very brief run in which insufficient time may have elapsed for diffusion to have provided enough helium to observe.

Our interest was aroused and has since been maintained, in spite of the appearance of much more complete tables of packing effects by Aston showing the unlikelihood of such results from energy considerations, by the statement of Riding and Baly to the effect that in the presence of a magnesium nitride film on their electrodes, helium was always obtained and neon generally, whereas these gases were never observed under conditions identical except for the absence of the film of nitride. No explanation of this particular phenomenon has been presented in the numerous criticisms which have appeared to date.

It seemed important, therefore, to attempt a repetition of the experiment. The procedure of Riding and Baly has been followed, introducing such alterations in the design of the apparatus as to make two identical bombarding and observation chambers differing only in the presence of a nitride film on the electrodes of one. Pyrex glass was used throughout, thus permitting a temperature of $450^{\circ} \mathrm{C}$. during the out-gassing process. Mereury diffusion pumps were employed to evacuate the apparatus, and diffusion pumps were substituted for the Sprengel pump used by Riding and Baly to transfer the supposed products of disintegration from one point to another. A highfrequency electrodeless discharge was used to excite the residual gases in the observation tubes instead of the discharge between electrodes as was the case in the earlier experiments.

Each experiment was preceded by a thorough evacuation and simultaneous out-gassing at $450^{\circ} \mathrm{C}$. extending from 24 to 48 hours, but the system was not washed out with hydrogen--which Paneth has found to be so essential. The apparatus was then allowed to stand for several days for minute leaks to be made evident. When no helium was found at the expiration of this period, the discharge was begun and continued for upwards of 100 hours at intermittent intervals. This part of the experiment extended usually for two weeks. In no case was any evidence found for the production of the noble gases from the nitride film, and it is our conviction that, as Paneth and others maintain, the appearance of such gases, helium particularly, is due to their diffusion in minute quantities from the atmosphere.

Colton Laboratory,

Donald Dooley.

Hiram College, Ohio, July 15.

\section{Raman Spectrum and Fluorescence of Benzol.}

RECENT work on the Raman effect has indicated that the frequencies so determined may be attributed to vibrational frequencies, or combinations of such frequencies, of the normal electronic state of the molecule. It is therefore to be expected that these levels will appear as end states in the process of emission or fluorescence. This is neatly confirmed in the case of benzol.

Data on the fluorescence of benzol vapour is available from the work of Reimann (Ann. d. Phys., $80,43 ; 1926)$ and Marsh (J. Chem. Soc., 123, 3319; 1923); although neither set of data can claim a high degree of accuracy, there is a satisfactory agreement between the two. It has now been found that all of the 51 bands given by Reimann can be arranged in series according to the general equation :

$$
\nu=\nu_{0}+n^{\prime} a-n^{\prime \prime}{ }_{1} b_{1}-n^{\prime \prime}{ }_{2} b_{2}-n^{\prime \prime}{ }_{3} b_{3}-n^{\prime \prime}{ }_{4} b_{4},
$$

where $n^{\prime}$ and the $n^{\prime \prime}$ 's are the vibrational quantum numbers of the excited and normal states respectively, and $a, b_{1}, b_{2}, b_{3}$, and $b_{4}$ are the corresponding frequencies. The value of $a$ is taken from the work of Henri on the absorption spectrum of benzol (see his "Structure des Molecules" (1925), p. 110) and is equal to $921 \mathrm{~cm}^{-1}$. The $b$ levels, together with the corresponding Raman values in parentheses as averaged from recent literature, are :

$$
b_{1}=998(991), \quad b_{2}=600(605), \quad b_{3}=855(853),
$$

Finally, $\nu_{0}=37494$ is given by Henri as the first member of an $n^{\prime}$ progression in absorption.

The following equations represent all of Reimann's data :

$$
\begin{aligned}
\text { (1) } \nu= & 37494+n^{\prime}(921)-n^{\prime \prime}{ }_{1}(998) \\
& n^{\prime}=0,1,2,3 ; n^{\prime \prime}{ }_{1}=0,1,2,3,4,5,6,7 . \\
\text { (2) } \nu= & 37494+n^{\prime}(921)-n^{\prime \prime}{ }_{1}(998)-n^{\prime \prime}{ }_{2}(600) \\
& n^{\prime}=0,1,2 ; n^{\prime \prime}{ }_{1}=0,1,2,3,4 ; n^{\prime \prime}{ }_{2}=1 . \\
\text { (3) } \nu= & 37494+n^{\prime}(921)-n^{\prime \prime}{ }_{1}(998)-n^{\prime \prime}{ }_{3}(855) \\
& n^{\prime}=2 ; n^{\prime \prime}{ }_{1}=0,1,2,3 ; n^{\prime \prime}=1,2 . \\
\text { (4) } \nu= & 37494+n^{\prime}(921)-n^{\prime \prime}{ }_{1}(998)-n^{\prime \prime}{ }_{4}(1180) \\
& n^{\prime}=2,3 ; n^{\prime \prime}{ }_{1}=0,1,2,3,4,5,6 ; n^{\prime \prime}{ }_{4}=1,2 .
\end{aligned}
$$

A fact which strongly corroborates the above arrangement is that more than half of the fluorescence bands originate on the $n^{\prime}=2$ level, the wave-length of which in absorption, 2540 A., is very close to the intense line at $2537 \mathrm{~A}$. in the mercury arc, the source of illumination in the fluorescence experiments.

In view of the relatively low accuracy of the above data, it is not possible to calculate the quadratic terms which are to be expected for a non-harmonic vibrator, although experience indicates that this factor is relatively small for organic molecules. As it is, the agreement between observed and calculated values is quite satisfactory.

$$
\text { C. V. Shapiro }
$$

(National Research Fellow).

Physikalisch-Chemisches

Institut der Universität, Zürich, July 29.

No. 3123, VoL. 124] 